18,12

\title{
Термофорез углеродных наночастиц (нанолент и нанотрубок) на плоской многослойной подложке гексагонального нитрида бора (h-BN)
}

\author{
(C) А.В. Савин \\ ${ }^{1}$ Федеральный исследовательский центр химической физики им. Н.Н. Семенова РАН, \\ Москва Россия \\ ${ }^{2}$ Российский экономический университет им. Г.В. Плеханова, \\ Москва, Россия \\ E-mail: asavin@center.chph.ras.ru
}

Поступила в Редакцию 28 июля 2021 г.

В окончательной редакции 28 июля 2021 г.

Принята к публикации 8 августа 2021 г.

\begin{abstract}
Методом молекулярной динамики с использованием 2D цепной модели показано, что термофорез углеродных наночастиц (нанолент и нанотрубок) на плоской многослойной подложке (на плоской поверхности кристалла гексагонального нитрида бора) обладает высокой эффективностью. Помещение наночастицы на плоскую поверхность подложки, участвующей в теплопереносе, приводит к ее движению в направлении потока тепла. Тепловой поток вдоль подложки приводит к образованию действующих на узлы наночастицы постоянных сил (сил термофореза). Главное действие силы оказывают на края нанолент графена, именно здесь происходит основное взаимодействие наноленты с изгибными фононами подложки. Данные фононы обладают большой длиной свободного пробега, поэтому эффективный перенос наночастиц с использованием термофореза может происходить на достаточно больших расстояниях. Движение углеродных наночастиц под действием теплового потока имеет вид движения частиц в вязкой среде под действием постоянной силы. Со временем наночастицы всегда выходят на режим движения с постоянной скоростью. Скорость стационарного движения практически одинакова для всех размеров и типов углеродных наночастиц, что объясняется тем, что сила термофореза и эффективное трение имеют один и тот же источник - взаимодействие наночастицы с изгибными тепловыми колебаниями слоев подложки.
\end{abstract}

Ключевые слова: наноленты, нанотрубки, плоские многослойные подложки, термофорез, 2D-модель многослойной подложки.

DOI: $10.21883 /$ FTT.2021.12.51687.178

\section{1. Введение}

Термофорезом называется направленное движение частиц, вызванное наличием внешнего теплового градиента. В последнее время термофорез стал новым методом манипулирования наноразмерными частицами [1-3]. Двумерные (2D) слоистые материалы, такие как графен $(\mathrm{G})$, гексагональный нитрид бора (h-BN), дисульфид молибдена $\left(\mathrm{MoS}_{2}\right)$ и вольфрама $\left(\mathrm{WS}_{2}\right)$, обладающие хорошей теплопроводностью и низким поверхностным трением, могут быть использованы в качестве платформы для транспортировки тел наноразмера [4].

Моделирование направленного движения наночастиц на плоской поверхности листа графена проводилось во многих работах. Так моделирование движения кластера атомов золота $\mathrm{Au}_{459}$ показало, что на плоской поверхности графита кластер может демонстрировать „баллистический“ высокоскоростной режим динамики с вязким трением [5]. Моделирование движения кластера по листу графена под действием теплового потока [6] показало, что кластер обладает баллистическим режимом направленного движения, который обусловлен взаимодействием кластера с изгибными фононами листа.
Анализ движения молекулы фуллерена $\mathrm{C}_{60}$ и коротких углеродных нанотрубок на листе графена $[7,8]$ показал наличие двух режимов термофореза: диффузионного (при низких температурах) и баллистического (при высоких температурах). Моделирование переноса нанопластинок графена по листу графена проведено в [9]. Показано, что градиент температуры играет ключевую роль в эволюции движения нанопластинки. Симуляция динамики нанокапли воды $\left(\mathrm{H}_{2} \mathrm{O}\right)_{1200}$ по поверхности листа графена и гексагонального нитрида бора (h-BN) показала [10], что взаимодействие капли с тепловым потоком приводит к ее направленному движению. Капля более сильно взаимодействует с поверхностью листа h-BN и двигается по нему более быстро. В работе [11] показано, что быстрая динамика частиц на плоской поверхности может быть связана с тем, что частица может двигаться по слою графена синхронно с бегущей изгибной поверхностной волной, оставаясь постоянно в яме этой волны.

Явление термофореза также активно моделировалось для наночастиц, находящихся внутри углеродных нанотрубок: для отдельных атомов [12], кластеров ато- 


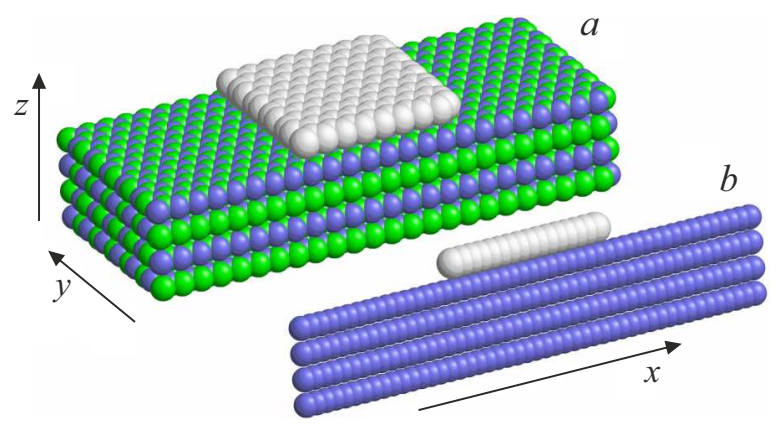

Рис. 1. Построение 2D цепной модели наноленты графена, лежащей на плоской поверхности кристалла h-BN. Показан (a) прямоугольный лист графена размера $2.02 \times 1.88 \mathrm{~nm}^{2}$ (160 атомов углерода), лежащий на плоской многослойной h-BN подложке, и $(b)$ соответствующая двухмерная цепная модель системы.

мов $[13,14]$, нанокапель воды [15-20], вложенных нанотрубок [21-23] и молекул фуллерена $\mathrm{C}_{60}$ [24,25]. Взаимодействие этих наночастиц с потоком тепловых фонов всегда приводит к их направленному движению от теплого конца нанотрубки к холодному.

Целью настоящей работы является численное моделирование термофореза углеродных наночастиц (нанолент и нанотрубок) на плоской многослойной подложке гексагонального нитрида бора (h-BN) - cм. рис. 1. Будет показано, что помещение наночастицы на плоскую многослойную подложку, участвующую в теплопереносе, приводит к ее движению с постоянной скоростью $v_{s}$ в направлении теплопереноса. Величина этой скорости практически одинакова для всех размеров и типов углеродных наночастиц. Динамика имеет вид баллистического движения частиц в вязкой среде под действием постоянной силы. Здесь сила термофореза и эффективное трение имеют один и тот же источник взаимодействие наночастицы с изгибными тепловыми колебаниями слоев подложки.

Работа построена следующим образом. В разд. 2 строится 2D цепная модель, используемая далее для моделирования движения углеродных наночастиц (нанолент и нанотрубок) по плоской многослойной подложке h-BN. B разд. 3 проводится моделирование торможения свободного движения наночастиц в результате их взаимодействия с термализованной подложкой. В разд. 4 определяется распределение вдоль наночастицы сил термофореза, обусловленных взаимодействием атомов наночастицы с тепловым потоком, идущим вдоль подложки. В разд. 5 проводится моделирование движения наночастиц под действием потока. Заключение приводится в разд. 6.

\section{2. Модель}

Для описания динамики углеродных наночастиц (нанолент и нанотрубок) на плоской поверхности мно- гослойной подложки удобно использовать 2D-модель системы молекулярных цепей [26-28]. Рассмотрим в качестве подложки плоскую поверхность кристалла h-BN. Будем считать, что наночастица и листы h-BN подложки лежат так, что у них направление зигзаг совпадает с осью $x-$ см. рис. 1. В этом случае двухмерная цепная модель будет описывать сечение системы наночастица + многослойная подложска вдоль оси $x$. Одному узлу в модели будут соответствовать все атомы наночастицы (слоя подложки), имеющие одинаковые координаты $x, z$.

Если атомы, расположенные вдоль одной линии параллельной оси $y$, двигаются синхронно, меняя только координаты $x, z$, то гамильтониан одной наноленты (нанотрубки) графена (h-BN) будет иметь вид гамильтониана цепи, расположенной в плоскости $x z$ :

$$
H_{i}=\sum_{n=1}^{N} \frac{1}{2} M_{i}\left(\dot{\mathbf{u}}_{n}, \dot{\mathbf{u}}_{n}\right)+\sum_{n=1}^{N-1} V_{i}\left(R_{n}\right)+\sum_{n=2}^{N-1} U_{i}\left(\theta_{n}\right)
$$

где индекс $i=1$, если рассматривается нанолента графена $(\mathrm{G})$, и $i=2$, если рассматривается нанолента бор нитрида $(\mathrm{BN})$. Двумерный вектор $\mathbf{u}_{n}=\left(x_{n}, z_{n}\right)$ задает координаты $n$-ой частицы цепи. Масса частицы для цепи $\mathrm{G}$ совпадает с массой атома углерода $M_{1}=M_{\mathrm{C}}=12 m_{p}$, а для цепи $\mathrm{BN}-$ со средней массой атомов бора и нитрида $M_{2}=\left(M_{\mathrm{B}}+M_{\mathrm{N}}\right) / 2=12.4085 m_{p}$ $\left(m_{p}=1.6603 \cdot 10^{-27} \mathrm{~kg}\right.$ - масса протона).

Потенциал

$$
V_{i}(R)=\frac{1}{2} K_{i}\left(R-R_{i}\right)^{2},
$$

описывает продольную жесткость цепи, $K_{i}$ - жесткость взаимодействия, $R_{i}$ - равновесная длина „валентной“ связи (шаг цепи), $R_{n}=\left|\mathbf{u}_{n+1}-\mathbf{u}_{n}\right|-$ расстояние между соседними узлами $n$ и $n+1$.

Потенциал

$$
U_{i}(\theta)=\epsilon_{i}[1+\cos (\theta)],
$$

описывает изгибную жесткость цепи, $\theta-$ угол между двумя соседними „валентными“ связями,

$$
\cos \left(\theta_{n}\right)=-\left(\mathbf{v}_{n-1}, \mathbf{v}_{n}\right) / R_{n-1} R_{n}, \text { вектор } \mathbf{v}_{n}=\mathbf{u}_{n+1}-\mathbf{u}_{n} .
$$

Параметры потенциалов (2), (3) для цепи G определены в [26,27] из анализа дисперсионных кривых наноленты графена. Продольная жесткость $K_{1}=405 \mathrm{~N} / \mathrm{m}$, шаг цепи $R_{1}=r_{C C} \sqrt{3} / 2=1.228 \AA \quad\left(r_{C C}=1.418 \AA-\right.$ длина валентной связи $\mathrm{C}-\mathrm{C}$ в листе графена), энергия $\epsilon_{1}=3.5 \mathrm{eV}$.

Нанолента h-BN имеет такую же структуру, как и нанолента графена. В двухмерной модели ее гамильтониан тоже будет иметь вид (1). Для нахождения параметров цепи BN была рассмотрена нанолента h-BN. Взаимодействие атомов наноленты описывалось расширенным потенциалом Терсоффа для нитрида бора [29]. Вычисления показали, что нанолента h-BN имеет такую 
же гексагональную структуру, как и нанолента графена. В основном состоянии длина валентной связи В-N $r_{B N}=1.445685 \AA$ незначительно превосходит длину валентной связи $\mathrm{C}-\mathrm{C}$. Дисперсионные кривые двумерной цепи с гамильтонианом (1) наилучшим образом соответствуют дисперсионным кривым полноатомной модели наноленты при жесткости $K_{2}=480 \mathrm{~N} / \mathrm{m}$, шаге цепи $R_{2}=r_{B N} \sqrt{3} / 2=1.252 \AA$ и энергии $\epsilon_{2}=1.10 \mathrm{eV}$.

Гамильтониан цепи (1) дает энергию наноленты, приходящуюся на продольную полосу ширины $\Delta y=R_{i} / \sqrt{3}$. Поэтому, если энергию системы цепей далее будем нормировать по наноленте графена, то энергию нанолент h-BN нужно умножить на нормирующий множитель $c=R_{1} / R_{2}=0.9808$.

Для вычисления эффективного потенциала невалентного взаимодействия узлов цепей (энергии взаимодействия одного атома с поперечной линией атомов) были использованы потенциалы Леннарда-Джонса $(6,12)$

$$
V(r)=\epsilon_{0}\left[\left(r_{0} / r\right)^{12}-2\left(r_{0} / r\right)^{6}\right],
$$

со значениями энергии $\epsilon_{0}$ и равновесными длинами взаимодействия $r_{0}$, представленными в табл. 1 .

Проведенные вычисления показали, что взаимодействия узлов цепей, соответствующих слоям бор нитридной подложки и слою графена, можно с высокой точностью описать потенциалом Леннарда-Джонса $(5,11)$

$$
W_{i}(r)=\varepsilon_{i}\left[5\left(r_{i} / r\right)^{11}-11\left(r_{i} / r\right)^{5}\right] / 6,
$$

где $r-$ расстояние между взаимодействующими узлами (индекс $i=1$, если описывается взаимодействие узлов цепей $\mathrm{BN}, i=2$ - взаимодействие узлов цепей $\mathrm{BN}$ и $\mathrm{G}$ ). Энергия взаимодействия $\varepsilon_{1}=0.01511, \varepsilon_{2}=0.01433 \mathrm{eV}$, равновесные расстояния $r_{1}=3.642, r_{2}=3.701 \AA$.

При моделировании динамики многослойной подложки необходимо ограничить число слоев. Поэтому будем считать, что первый (самый нижний) слой уже взаимодействует с неподвижной плоской поверхностью кристалла (на рис. 2 эта поверхность показана черной линией).

Вычисления показывают, что потенциал взаимодействия с неподвижной подложкой $W_{0}(h)$ (зависимость энергии узла цепи от расстояния $h$ до плоскости подложки) с высокой точностью может быть описана $(k, l)$ потенциалом Леннарда-Джонса

$$
W_{0}(h)=\varepsilon_{0}\left[k\left(h_{0} / h\right)^{l}-l\left(h_{0} / h\right)^{k}\right] /(l-k),
$$

где степень $l=10, k=3.75$, энергия взаимодействия $\varepsilon_{0}=0.0974 \mathrm{eV}$, равновесное расстояние $h_{0}=3.49 \AA$.

Таблица 1. Значения параметров потенциала ЛеннардаДжонса (4) для различных пар взаимодействующих атомов [30].

\begin{tabular}{l|c|c|c|c|c}
\hline & $\mathrm{BB}$ & $\mathrm{NN}$ & $\mathrm{BN}$ & $\mathrm{CB}$ & $\mathrm{CN}$ \\
\cline { 2 - 6 }$\epsilon_{0}(\mathrm{meV})$ & 7.81 & 2.99 & 4.81 & 5.96 & 3.69 \\
$r_{0}(\AA)$ & 4.083 & 3.660 & 3.866 & 3.976 & 3.756
\end{tabular}

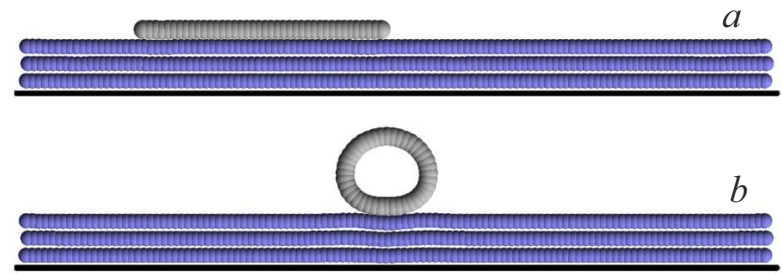

Рис. 2. Вид стационарного состояния $(a)$ однослойного листа графена длины $L=4.79 \mathrm{~nm}$ (число звеньев $\mathrm{G}$ цепи $N_{c}=40$ ) и $(b)$ однослойной нанотрубки с индексом хиральности $(20,0)$ (число звеньев циклической цепи $N_{c}=40$ ) на многослойной подложке, образованной поверхностью кристалла h-BN (число слоев подложки $K-1=3$, число звеньев в $\mathrm{BN}$ цепи $N_{b n}=360$. Черная прямая показывает положение поверхности неподвижной подложки.

Рассмотрим $K$-слойные структуры, представленные на рис. 2. Пусть первые $k=1, \ldots, K-1$ слоев соответствуют BN цепям (слоям кристалла h-BN), состоящим из $N_{b n}$ звеньев. Эти слои лежат на плоской твердой подложке и взаимодействуют с ней (будем считать, что поверхность твердой подложки совпадает с плоскостью $z=0)$. Последняя $K$-тая $\mathrm{G}$ цепь, имеющая $N_{c} \ll N_{b n}$ звеньев, соответствует углеродной наночастице (наноленте, нанотрубке), лежащей на плоской деформируемой многослойной подложке. Координаты узлов этой системы $K$ цепей задаются векторами $\left\{\mathbf{u}_{n, k}=\left(x_{n, k}, z_{n, k}\right)\right\}_{n=1, k=1}^{N_{k}, K}$, где $N_{k}$ - число узлов в $k$-той цепи $\left(N_{k}=N_{b n}\right.$ при $k=1, K-1$ и $\left.N_{K}=N_{c}\right)$.

Гамильтониан системы цепей будет иметь вид

$$
\begin{aligned}
H= & \sum_{k=1}^{K-1} \sum_{n=1}^{N_{b n}} \frac{1}{2} c M_{2}\left(\dot{\mathbf{u}}_{n, k}, \dot{\mathbf{u}}_{n, k}\right) \\
& +\sum_{n=1}^{N_{c}} \frac{1}{2} M_{1}\left(\dot{\mathbf{u}}_{n, K}, \dot{\mathbf{u}}_{n, K}\right)+E,
\end{aligned}
$$

где потенциальная энергия

$$
\begin{aligned}
E= & c \sum_{k=1}^{K-1}\left[\sum_{n=1}^{N_{b n}-1} V_{2}\left(R_{n, k}\right)\right. \\
& \left.+\sum_{n=2}^{N_{b n}-1} U_{2}\left(\theta_{n, k}\right)+\sum_{n=1}^{N_{b n}} W_{0}\left(z_{n, k}\right)\right] \\
& +c \sum_{k_{1}=1}^{K-2} \sum_{k_{2}=k_{1}+1}^{K-1} \sum_{n=1}^{N_{b n}} \sum_{l=1}^{N_{b n}} W_{1}\left(r_{n, k_{1} ; l, k_{2}}\right) \\
& +\sum_{n=1}^{N_{c}-1} V_{1}\left(R_{n, K}\right)+\sum_{n=2}^{N_{c}-1} U_{1}\left(\theta_{n, K}\right) \\
& +\sum_{k=1}^{K-1} \sum_{n=1}^{N_{b n}} \sum_{l=1}^{N_{c}} W_{2}\left(r_{n, k ; l, K}\right) .
\end{aligned}
$$


Здесь расстояние между соседними узлами $k$-той цепи $R_{n, k}=\left|\mathbf{u}_{n+1, k}-\mathbf{u}_{n, k}\right|$, косинус угла между двумя соседними связями

$$
\cos \left(\theta_{n, k}\right)=-\left(\mathbf{v}_{n-1, k}, \mathbf{v}_{n, k}\right) / R_{n-1, k} R_{n, k},
$$

вектор $\mathbf{v}_{n, k}=\mathbf{u}_{n+1, k}-\mathbf{u}_{n, k}$, расстояние между узлами разных цепей $k_{1}$ и $k_{2} r_{n, k_{1} ; l, k_{2}}=\left|\mathbf{u}_{l, k_{2}}-\mathbf{u}_{n, k_{1}}\right|$. В формуле потенциальной энергии первое слагаемое (8) задает энергию деформации $\mathrm{BN}$ цепей с учетом энергии их взаимодействия с твердой подложкой, второе слагаемое (9) - энергию невалентного взаимодействия BN цепей, третье слагаемое (10) - энергию деформации $\mathrm{G}$ цепи, а последнее слагаемое (11) - энергию невалентного взаимодействия $\mathrm{G}$ цепи с $\mathrm{BN}$ цепями.

\section{3. Движение наночастиц на термализованной подложке}

Рассмотрим систему, состоящую из $K-1=3$ слоев $\mathrm{BN}$ цепей с $N_{b n}=2400$ звеньями. Длина такой трехслойной подложки $L=\left(N_{b n}-1\right) R_{2}=300.4 \mathrm{~nm}$. В качестве углеродной наночастицы, расположенной на этой подложке, возьмем линейную (циклическую) $\mathrm{G}$ цепочку из $N_{c}$ звеньев.

Поместим сначала наночастицу у левого края подложки, как показано на рис. 2. Далее найдем стационарное состояние системы „наночастица + трехслойная подложка“. Для этого численно методом сопряженных градиентов решим задачу на минимум энергии

$$
E \rightarrow \min :\left\{\mathbf{u}_{n, k}\right\}_{n=1, k=1}^{N_{k}, K},
$$

где число цепей $K=4$, число узлов $N_{1}=N_{2}=N_{3}=N_{b n}$ $=2400, N_{4}=N_{c}$. Типичный вид стационарных состояний показан на рис. 2.

Для получения термализованного состояния системы поместим ее в термостат Ланжевена. Для этого зафиксируем $x$ координаты краевых частиц $\mathrm{BN}$ цепей (положим скорости $\left.\left\{\dot{x}_{1, k} \equiv 0, \dot{x}_{N_{b n}, k} \equiv 0\right\}_{k=1}^{K-1}\right)$ и $x$ координату центральной частицы $\mathrm{G}$ цепи $\left(\dot{x}_{N_{c} / 2, K} \equiv 0\right)$ и численно проинтегрируем систему уравнений Ланжевена

$$
\begin{gathered}
M_{2} \ddot{\mathbf{u}}_{n, k}=-\frac{1}{c} \frac{\partial H}{\partial \mathbf{u}_{n, k}}-\Gamma M_{2} \dot{\mathbf{u}}_{n, k}+\Xi_{n, k}, \\
k=1, \ldots, K-1, n=1, \ldots, N_{b n}, \\
M_{1} \ddot{\mathbf{u}}_{n, K}=-\frac{\partial H}{\partial \mathbf{u}_{n, K}}-\Gamma M_{1} \dot{\mathbf{u}}_{n, K}+\Xi_{n, K}, \\
n=1, \ldots, N_{c},
\end{gathered}
$$

где $\Gamma=1 / t_{r}-$ коэффициент трения (время релаксации $\left.t_{r}=0.4 \mathrm{ps}\right), \Xi_{n, k}=\left(\xi_{n, k, 1}, \xi_{n, k, 2}\right)$ - двухмерный вектор нормально распределенных случайных сил, нормализованных условиями

$$
\left\langle\xi_{n, k, i}\left(t_{1}\right) \xi_{m, l, j}\left(t_{2}\right)\right\rangle=2 M \Gamma k_{B} T \delta_{n m} \delta_{k l} \delta_{i j} \delta\left(t_{2}-t_{1}\right)
$$

( $T$ - температура термостата, $k_{B}$ - постоянная Больцмана, масса $M=M_{2} / c$ при $k<K$ и $M=M_{1}$ при $\left.k=K\right)$.
Возьмем начальные условия для системы уравнений движения Ланжевена (13), (14) соответствующие основному стационарному состоянию системы $\left\{\mathbf{u}_{n, k}(0)=\mathbf{u}_{n, k}^{0}, \quad \dot{\mathbf{u}}_{n, k}(0)=\mathbf{0}\right\}_{n=1, k=1}^{N_{k}, K}$ и численно проинтегрируем систему в течение времени $t_{0}=20 t_{r}$. За это время система цепей придет в полное равновесие с термостатом, и мы получим ее термализованное состояние $\left\{\mathbf{w}_{n, k}=\mathbf{u}_{n, k}\left(t_{0}\right), \mathbf{v}_{n, k}=\dot{\mathbf{u}}_{n, k}\left(t_{0}\right)\right\}_{n=1, k=1}^{N_{k}, K}$.

Для моделирования свободного движения наночастицы по термализованной многослойной подложке оставим фиксированными только $x$ координаты краевых узлов BN цепей, уберем взаимодействие системы с термостатом, а всем атомам наночастицы сообщим дополнительную начальную скорость $v_{0}>0$, направленную вдоль оси $x$. Для этого численно проинтегрируем систему уравнений движения

$$
\begin{gathered}
M_{2} \dot{\mathbf{u}}_{n, k}=-\frac{1}{c} \frac{\partial H}{\partial \mathbf{u}_{n, k}}, \\
k=1, \ldots, K-1, \quad n=1, \ldots, N_{b n}, \\
M_{1} \ddot{\mathbf{u}}_{n, K}=-\frac{\partial H}{\partial \mathbf{u}_{n, K}}, \quad n=1, \ldots, N_{c},
\end{gathered}
$$

с начальными условиями

$$
\begin{gathered}
\mathbf{u}_{n, k}(0)=\mathbf{w}_{n, k}, \quad \dot{\mathbf{u}}_{n, k}(0)=\mathbf{v}_{n, k}, \\
n=1, \ldots, N_{b n}, \quad k=1, \ldots, K-1, \\
\mathbf{u}_{n, K}(0)=\mathbf{w}_{n, K}, \dot{\mathbf{u}}_{n, K}(0)=\mathbf{v}_{n, K}+v_{0} \mathbf{e}_{x}, \\
n=1, \ldots, N_{c},
\end{gathered}
$$

где вектор $\mathbf{e}_{x}=(1,0)$.

Проследим за движением центра тяжести наночастицы $x_{c}=\left(x_{1, K}+x_{2, K}+\ldots+x_{N_{c}, K}\right) / N_{c}$ вдоль подложки. Характер движения углеродных наночастиц (нанолент и нанотрубок) показан на рис. 3. Как видно из рисунка, вид траектории $x_{c}(t)$ зависит от реализации начального термализованного состояния системы, но взаимодействие с подложкой всегда приводит к торможению направленного движения частицы (начальная скорость движения $v_{0}=500 \mathrm{~m} / \mathrm{s}$ ). Если траектории усреднить по всем независимым реализациям термализованного состояния системы (взять среднее значение $\left.\bar{x}_{c}(t)=\left\langle x_{c}(t)\right\rangle\right)$, то динамика центра тяжести наночастицы с хорошей точностью описывается как движение частицы в вязкой среде

$$
\bar{x}_{c}(t)=\bar{x}_{c}(0)+v_{0}[1-\exp (-\gamma t)] / \gamma,
$$

с коэффициентом вязкого „трения“ $\gamma>0$ (обратная величина $\gamma^{-1}$ соответствует времени, за которое начальная скорость наночастицы уменьшается в $e$ раз). Так при $N_{c}=40$ и температуре $T=300 \mathrm{~K}$ коэффициент эффективного трения для движения по подложке наноленты и нанотрубки $\gamma=4.32$ и $4.56 \mathrm{~ns}^{-1}-$ см. рис. $3(a)$ и $(b)$.

Численное моделирование динамики наночастиц показало, что ее вязкое торможение (19) происходит при всех размерах наночастиц и температурах подложки 


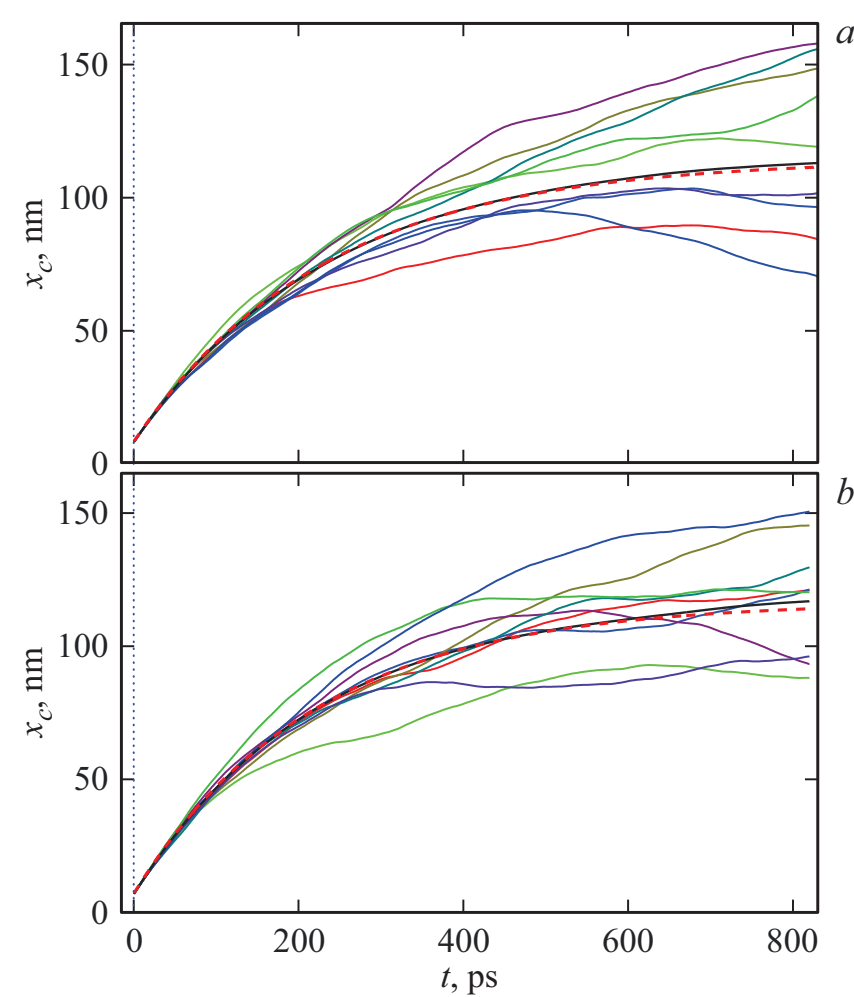

Рис. 3. Зависимость от времени $t$ центра тяжести углеродной наночастицы $x_{c}$ при температуре подложки $T=300 \mathrm{~K}$. Тонкие кривые показывают 9 траекторий движения для различных независимых начальных реализаций термализованного состояния системы. Сплошная толстая (черная) линия дает траекторию среднего значения центра тяжести $\bar{x}_{c}$, полученную по 256 независимым реализациям термализованного состояния. Часть $(a)$ показывает динамику для линейной цепи (наноленты), часть $(b)$ - для циклической цепи (нанотрубки) с $N_{c}=40$ звеньями, начальная скорость движения наночастицы $v_{0}=500 \mathrm{~m} / \mathrm{s}$. Пунктирные (красные) линии показывают зависимость (19) при значении коэффициента эффективного трения $\gamma=4.32$ и $4.56 \mathrm{~ns}^{-1}$.

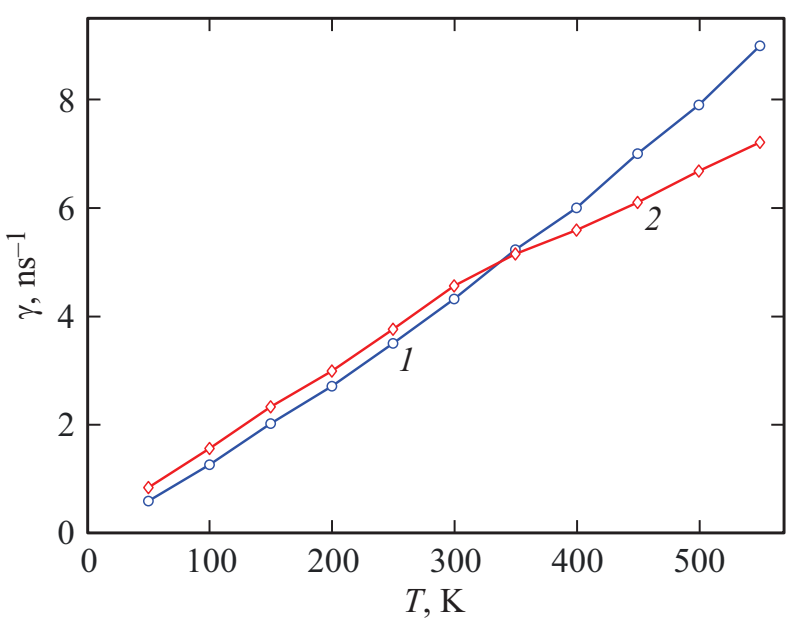

Рис. 4. Зависимость от температуры $T$ коэффициента трения $\gamma$ для линейной (наноленты) и циклической цепи (нанотрубки) из $N_{c}=40$ звеньев (кривая 1 и 2).
$50 \leq T \leq 550 \mathrm{~K}$, но значение коэффициента эффективного трения $\gamma$ зависит от температуры, размера и типа наночастицы. Зависимость $\gamma$ от температуры показана на рис. 4. Как видно из рисунка, коэффициент трения монотонно растет с увеличением температуры. При $T \leq 300 \mathrm{~K}$ уменьшение температуры приводит к практически линейному уменьшению эффективного трения $\gamma$ до значений, близких к нулю. Это позволяет заключить, что причиной торможения наночастиц является их взаимодействие с тепловыми колебаниями. Главную роль в этом взаимодействии играют изгибные (поперечные) колебания цепей - если уменьшить их амплитуду, то значительно уменьшается и эффективное трение.

Заметим, что полученный существенный рост трения с увеличением температуры парадоксальным образом отличается от стандартных сценариев трения, где оно всегда уменьшается с увеличением температуры [31]. Отличие связано с тем, что в рассматриваемой системе трение имеет волновую природу (обусловлено взаимодействием с тепловыми колебаниями). Увеличение силы трения слоев G/h-BN с увеличением температуры также получено в работе [32].

\section{4. Сила термофореза}

Для прямого моделирования теплопереноса поместим первые $N_{t}=40$ узлов цепей подложки в термостат Ланжевена температуры $T_{+}$, а последние - в термостат температуры $T_{-}$(разница температуры $\left.\Delta T=T_{+}-T_{-} \geq 0\right)$. Тогда для моделирования теплопереноса вдоль подложки нужно численно проинтегрировать систему уравнений:

$$
\begin{gathered}
M_{2} \ddot{\mathbf{u}}_{n, k}=-\frac{1}{c} \frac{\partial H}{\partial \mathbf{u}_{n, k}}-\Gamma M_{2} \dot{\mathbf{u}}_{n, k}+\Xi_{n, k}^{+}, \quad n=1, \ldots, N_{t}, \\
M_{2} \ddot{\mathbf{u}}_{n, k}=-\frac{1}{c} \frac{\partial H}{\partial \mathbf{u}_{n, k}}, \quad n=N_{t}+1, \ldots, N_{b n}-N_{t}, \quad(20) \\
M_{2} \ddot{\mathbf{u}}_{n, k}=-\frac{1}{c} \frac{\partial H}{\partial \mathbf{u}_{n, k}}-\Gamma M_{2} \dot{\mathbf{u}}_{n, k}+\Xi_{n, k}^{-}, \\
n=N_{b n}-N_{t}+1, \ldots, N_{b n}, \quad k=1, \ldots, K-1, \\
M_{1} \ddot{\mathbf{u}}_{n, K}=-\frac{\partial H}{\partial \mathbf{u}_{n, K}}, \quad n=1, \ldots, N_{c},
\end{gathered}
$$

где $\Gamma=1 / t_{r}$ - коэффициент трения (время релаксации $\left.t_{r}=0.4 \mathrm{ps}\right), \Xi_{n, k}^{ \pm}=\left(\xi_{n, k, 1}^{ \pm}, \xi_{n, k, 2}^{ \pm}\right)$- двумерный вектор нормально распределенных случайных сил, нормализованных условиями

$$
\left\langle\xi_{n, k, i}^{ \pm}\left(t_{1}\right) \xi_{m, l, j}^{ \pm}\left(t_{2}\right)\right\rangle=2 M_{2} \Gamma k_{B} T_{ \pm} \delta_{n m} \delta_{k l} \delta_{i j} \delta\left(t_{2}-t_{1}\right) .
$$

Возьмем начальные условия для системы уравнений движения (20), соответствующие основному состоянию многослойной системы с расположением цепи графена по центру подложки. Зафиксируем $x$ координату центральной частицы цепи $\mathrm{G}$ (положим $\dot{x}_{N_{c} / 2, K} \equiv 0$ ) и численно проинтегрируем систему уравнений движения 


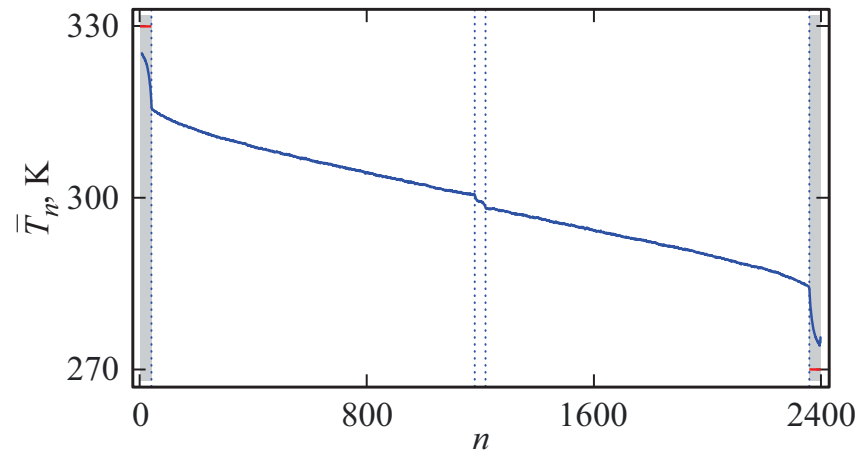

Рис. 5. Распределение вдоль трехслойной подложки локальной температуры $\bar{T}_{n}$. Каждая цепочка подложки состоит из $N_{b n}=2400$ звеньев. Температура краевых термостатов $T_{ \pm}=300 \pm 30 \mathrm{~K}$, число краевых звеньев, взаимодействующих с термостатами $N_{t}=40$. Серым цветом показаны краевые участки подложки, взаимодействующие с термостатами. Искажение температурного профиля в центре подложки связано с наличием $\mathrm{G}$ цепи из $N_{c}=40$ звеньев.

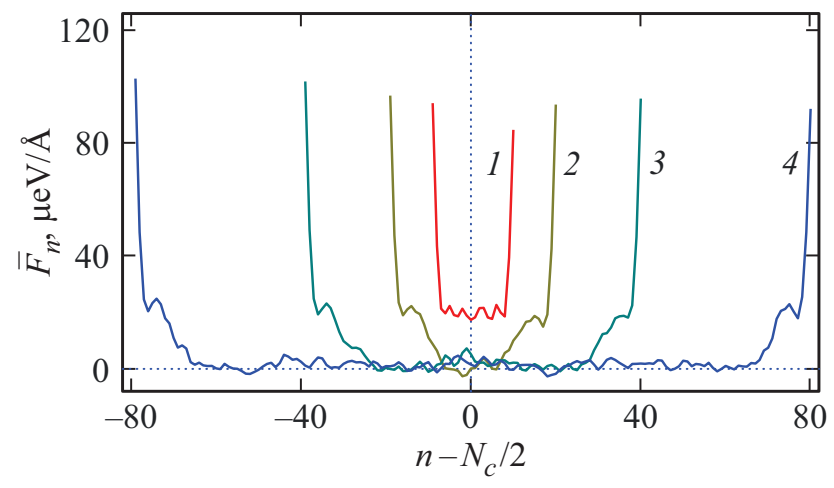

Рис. 6. Распределение вдоль $\mathrm{G}$ цепи сил термофореза $\bar{F}_{n}$ при числе звеньев цепи $N_{c}=20,40,80,160$ (кривые 1,2,3,4). Каждая цепочка подложки состоит из $N_{b n}=2400$ звеньев, температура краевых термостатов $T_{ \pm}=300 \pm 30 \mathrm{~K}$.

до образования стационарного теплового потока. В центральной части подложки $N_{t}<n \leq N_{b n}-N_{t}$ произойдет образование постоянного температурного градиента см. рис. 5. Распределение средних значений температуры вдоль подложки находилось по формулам:

$$
\bar{T}_{n}=\left\langle T_{n}\right\rangle=\lim _{t \rightarrow \infty} \frac{M_{2}}{2(K-1) k_{B} t} \int_{0}^{t} \sum_{k=1}^{K-1}\left|\dot{\mathbf{u}}_{n, k}(\tau)\right|^{2} d \tau .
$$

Найдем также средние значения продольных сил $F_{n, 1}$, действующих со стороны подложки на узлы $G$ цепи. Полный вектор силы

$$
\mathbf{F}_{n}=\left(F_{n, 1}, F_{n, 2}\right)=\sum_{k=1}^{K-1} \sum_{l=1}^{N_{b n}} \frac{\partial W_{2}\left(r_{l, k, n, K}\right)}{\partial \mathbf{u}_{n, K}},
$$

где расстояние $r_{l, k ; n, K}=\left|\mathbf{u}_{n, K}-\mathbf{u}_{l, k}\right|$.
Пусть

$$
\bar{F}_{n, 1}=\lim _{t \rightarrow \infty} \frac{1}{t} \int_{0}^{t} F_{n, 1}(\tau) d \tau
$$

- среднее значение продольной силы, действующей со стороны подложки на узел $n$ цепи $\mathrm{G}$ при наличии теплового потока, а $\bar{F}_{n, 1}^{0}-$ среднее значение силы при отсутствии теплового потока (при $T_{ \pm}=T$ ). Тогда наличие теплового потока будет приводить к образованию дополнительных сил $\bar{F}_{n}=\bar{F}_{n, 1}-\bar{F}_{n, 1}^{0}>0$, действующих на узлы $\mathrm{G}$ цепи в направлении потока.

Распределение сил термофореза $\bar{F}_{n}$ вдоль $\mathrm{G}$ цепи (вдоль наноленты графена) показано на рис. 6. Как видно из рисунка, главное действие силы термофореза оказывают на края наноленты, где изгибные колебания подложки из теплового потока фононов входят под наноленту графена и выходят из-под нее. Заметим, что увеличение изгибной жесткости цепей подложки приводит к существенному уменьшению сил термофореза, что говорит о главном вкладе в силы термофореза взаимодействия наноленты графена с изгибными колебаниями подложки.

Определим общую $F=\sum_{n=1}^{N_{c}} \bar{F}_{n}$, центральную $F_{c}=$ $=\sum_{n=16}^{N_{c}-15} \bar{F}_{n}$ и краевую силу термофореза $F_{e}=F-F_{c}$. Зависимость сил термофореза от длины наноленты графена (от числа узлов $\mathrm{G}$ цепи $N_{c}$ ) приведена в табл. 2. Как видно из таблицы, общая сила термофореза монотонно увеличивается с увеличением длины наноленты. Главный вклад в термофорез дают силы, действующие на краевые 15 звеньев $\mathrm{G}$ цепи, а рост общей силы происходит, в основном, за счет увеличения взаимодействия с подложкой центральной части цепи.

Моделирование теплопереноса показало, что сила термофореза $F$ всегда прямо пропорциональна разнице температур $\Delta T-$ см. рис. 7. С другой стороны, увеличение расстояния между термостатами (увеличение длины подложки) не приводит к существенному уменьшению силы термофореза. Так для $\mathrm{G}$ цепи из $N_{c}=40$ звеньев при $T_{ \pm}=300 \pm 30 \mathrm{~K}$ сила термофореза $F=790$ при числе звеньев в цепях подложки $N_{b n}=300$, $F=830$ при $N_{b n}=600, \quad F=770$ при $N_{b n}=1200$ и $F=610 \mu \mathrm{eV} / \AA$ при $N_{b n}=2400$. Это связано с тем, что сила термофореза обеспечивается, главным образом, давлением на наноленту графена поперечных (изгибных)

Таблица 2. Зависимость общей $F$, краевой $F_{e}$ и центральной силы термофореза $F_{c}$ от числа звеньев $\mathrm{G}$ цепи $N_{c}$ (от длины наноленты графена) при длине подложки $L=300.4 \mathrm{~nm}$ $\left(N_{b n}=2400\right)$ и температуре $T_{ \pm}=300 \pm 30 \mathrm{~K}$

\begin{tabular}{c|r|r|r|r|r|r}
\hline$N_{c}$ & 20 & 40 & 80 & 160 & 320 & 640 \\
\hline$F\left(10^{-6} \mathrm{eV} / \AA\right)$ & 586 & 610 & 703 & 828 & 966 & 1186 \\
$F_{e}\left(10^{-6} \mathrm{eV} / \AA\right)$ & 586 & 609 & 641 & 661 & 699 & 712 \\
$F_{c}\left(10^{-6} \mathrm{eV} / \AA\right)$ & 0 & 1 & 62 & 166 & 267 & 356
\end{tabular}




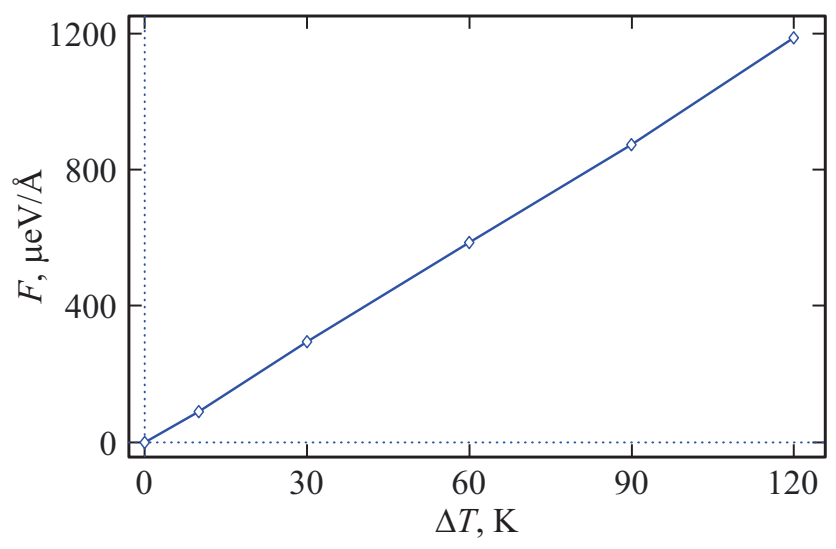

Рис. 7. Зависимость силы термофореза $F$ от разницы температур краевых термостатов $\Delta T$. Число звеньев $\mathrm{G}$ цепи $N_{c}=20$, число звеньев в каждой цепи подложки $N_{b n}=2400$, средняя температура подложки $T=300 \mathrm{~K}$.

длинноволновых фононов цепей подложки, а данные фононы обладают большой длиной свободного пробега. Поэтому эффективный перенос наночастиц с использованием термофореза может происходить на достаточно больших расстояниях.

\section{5. Движение наночастиц под действием теплового потока}

Проведенный анализ позволяет заключить, что движение наночастицы на плоской многослойной подложке h-BN может быть описано как ее движение в вязкой среде под действием постоянной силы

$$
M \ddot{x}_{c}=F-\gamma M \dot{x}_{c},
$$

где $M=N_{c} M_{1}-$ масса частицы, $x_{c}-x$ координата центра тяжести частицы. Из уравнения (21) следует, что со временем частица всегда будет двигаться с установившейся постоянной скоростью $v_{s}=F / \gamma M$.

Найдем скорость установившегося движения наночастицы $v_{s}$ из прямого численного моделирования динамики частицы по подложке, по которой происходит теплоперенос. Для этого численно проинтегрируем систему уравнений движения (20). Вначале расположим наночастицу у левого края подложки. Зафиксируем $x$

Таблица 3. Зависимость скорости установившегося движения $v_{s}$ наноленты (NR) и нанотрубки графена (NT) от числа звеньев цепи $N_{c}$ при длине подложки $L=300.4 \mathrm{~nm}$ $\left(N_{b n}=2400\right)$ и температуре $T_{ \pm}=300 \pm 30 \mathrm{~K}$

\begin{tabular}{c|c|c|c|c|c|c}
\hline \multirow{4}{*}{ NR } & $N_{c}$ & 20 & 40 & 80 & 160 & 320 \\
\cline { 2 - 7 } NT & $v_{s}(\mathrm{~m} / \mathrm{s})$ & 289 & 291 & 283 & 279 & 276 \\
$v_{s}(\mathrm{~m} / \mathrm{s})$ & 296 & 284 & 283 & 290 & 286
\end{tabular}

координату узла $N_{c} / 2$ цепи $\mathrm{G}$ и проинтегрируем систему до образования в подложке постоянного теплового потока. Затем сообщим всем узлам $\mathrm{G}$ цепи скорость $v_{s}$ и рассмотрим свободное движение цепи по подложке при наличии теплопереноса.

Численное моделирование показало, что под действием теплового потока наночастица на поверхности подложки всегда выходит на режим движения с постоянной скоростью $v_{s}>0-$ см. рис. 8. Конечно, вид траектории движения центра тяжести наночастицы $x_{c}(t)$ зависит от конкретной реализации термализованного состояния подложки. Но если усреднить по всем независимым реализациям начального термализованного состояния подложки, то движение центра тяжести будет происходить с постоянной скоростью:

$$
\bar{x}_{c}(t)=\left\langle x_{c}(t)\right\rangle=\bar{x}_{c}(0)+v_{s} t .
$$

Зависимость стационарной скорости движения $v_{s}$ от типа наночастицы и числа ее звеньев $N_{c}$ представлена в табл. 3. Как видно из таблицы, скорость движения

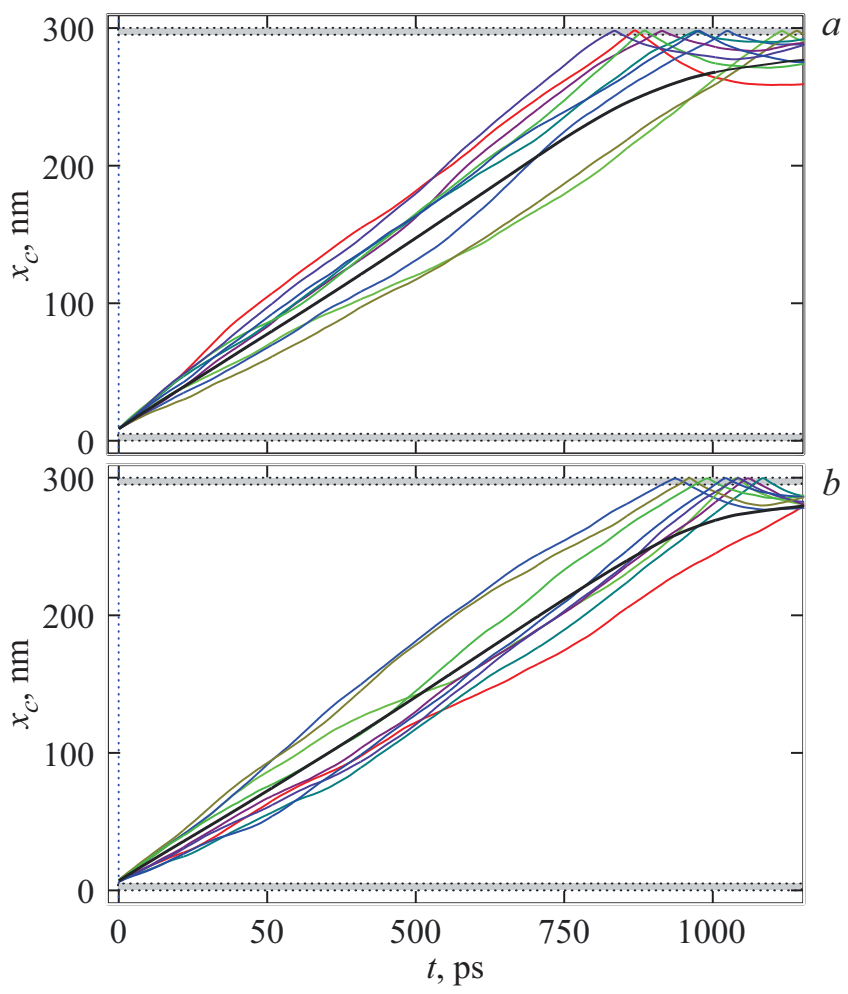

Рис. 8. Зависимость от времени $t$ центра тяжести углеродной наночастицы $x_{c}$ при температуре краевых термостатов $T_{ \pm}=300 \pm 30 \mathrm{~K}$ (серым цветом показаны края подложки, взаимодействующие с термостатами). Тонкие кривые показывают 9 траекторий движения для различных независимых начальных реализаций термализованного состояния подложки. Сплошная толстая (черная) линия дает траекторию среднего значения центра тяжести $\bar{x}_{c}$, полученную по 256 независимым реализациям термализованного состояния. Часть $(a)$ показывает динамику для линейной цепи (наноленты), часть $(b)-$ для циклической цепи (нанотрубки) с $N_{c}=40$ звеньями. 


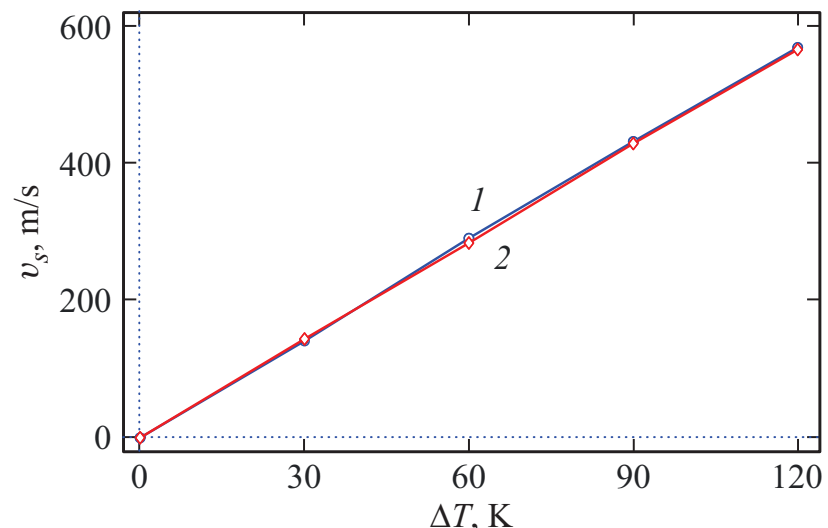

Рис. 9. Зависимость скорости установившегося движения наноленты и нанотрубки $v_{s}$ (кривая 1 и 2) от разницы температур $\Delta T$ (температура концевых термостатов $T_{ \pm}=T \pm \Delta T / 2$, $T=300 \mathrm{~K})$. Число звеньев $\mathrm{G}$ цепи $N_{c}=40$, число звеньев цепи подложки $N_{b n}=2400$.

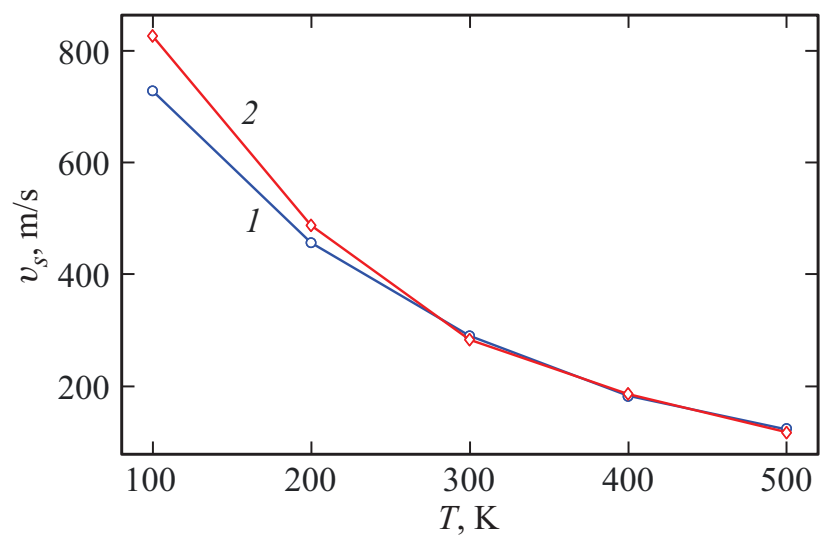

Рис. 10. Зависимость скорости установившегося движения наноленты и нанотрубки $v_{s}$ (кривая 1 и 2) от средней температуры подложки $T$. Число звеньев $\mathrm{G}$ цепи $N_{c}=40$, число звеньев цепи подложки $N_{b n}=2400$. Температура краевых термостатов $T_{ \pm}=T \pm 30 \mathrm{~K}$.

практически одинакова для всех типов наночастиц. Это говорит о том, что соотношение $F / \gamma M$ всегда остается почти одинаковым для всех углеродных наночастиц. Данный эффект обусловлен тем, что сила термофореза $F$ и общий коэффициент трения $\gamma$ имеют одинаковый источник - взаимодействие наночастицы с поперечными тепловыми колебаниями слоев подложки.

Линейная зависимость величины силы термофореза $F$ от разницы температур $\Delta T$ (рис. 7) обеспечивает также линейную зависимость скорости стационарного движения. Скорость движения $v_{s}$ всегда прямо пропорциональна разнице температур - см. рис. 9.

Монотонное уменьшение общего коэффициента трения $\gamma$ при уменьшении температуры подложки $T$ (рис. 4) приводит, при сохранении постоянной разницы температур $\Delta T$, к монотонному росту скорости стационарного движения наночастицы $v_{s}-$ см. рис. 10. Поэтому эффект термофореза углеродных наночастиц по плоской многослойной подложке h-BN будет наиболее сильно проявляться в области низких температур.

\section{6. Заключение}

Проведенное численное моделирование показывает, что термофорез углеродных наночастиц (нанолент и нанотрубок) на плоской многослойной подложке h-BN обладает высокой эффективностью. Под действием разницы температур на краях подложки $\Delta T$ в ней возникает постоянный тепловой поток, вызывающий направленное движение наночастиц от теплого края подложки к холодному.

Движение наночастиц на термализованной подложке с хорошей точностью описывается как движение частиц в вязкой среде. Моделирование динамики показало, что вязкое торможение происходит при всех размерах наночастиц и температурах подложки. Значение коэффициента эффективного трения частицы с подложкой $\gamma$ монотонно увеличивается с увеличением температуры. При $T \leq 300 \mathrm{~K}$ уменьшение температуры приводит к линейному уменьшению $\gamma$ до значений, близких к нулю. Причиной торможения наночастиц является их взаимодействие с тепловыми колебаниями подложки. Главную роль в этом взаимодействии играют изгибные (поперечные) колебания слоев подложки.

Тепловой поток вдоль подложки приводит к образованию действующих на узлы наночастицы постоянных сил (сил термофореза), направление которых совпадает с направлением теплопереноса. Вычисления показали, что главное действие силы оказывают на края наноленты графена, где изгибные колебания подложки из теплового потока фононов входят и выходят из-под нее. Общая сила термофореза $F$ монотонно увеличивается с увеличением длины наноленты. Главный вклад в нее дают силы, действующие на краевые 15 звеньев, а рост общей силы происходит за счет увеличения взаимодействия с подложкой центральной части цепи (наноленты).

Сила термофореза $F$ всегда прямо пропорциональна разнице температур $\Delta T$. При фиксированном значении $\Delta T$ увеличение расстояния между термостатами (увеличение длины подложки) не приводит к существенному уменьшению силы. Это связано с тем, что сила термофореза обеспечивается, главным образом, давлением на наноленту графена изгибных длинноволновых фононов слоев подложки, а данные фононы обладают большой длиной свободного пробега. В силу этого эффективный перенос наночастиц с использованием термофореза может происходить на достаточно больших расстояниях.

Движение наночастиц под действием теплового потока на плоской многослойной подложке h-BN может быть описано как их движение в вязкой среде под действием постоянной силы $F$. Поэтому со временем частица всегда будет двигаться в направлении теплового потока с постоянной скоростью $v_{s}$. Моделирование динамики 
показало, что скорость стационарного движения практически одинакова для всех размеров и типов углеродных наночастиц. Данный эффект обусловлен тем, что сила термофореза $F$ и общий коэффициент трения $\gamma$ имеют один и тот же источник - взаимодействие наночастицы с поперечными тепловыми колебаниями слоев подложки.

Величина скорости $v_{s}$ всегда прямо пропорциональна разнице температур. Монотонное уменьшение общего коэффициента трения $\gamma$ при уменьшении температуры подложки $T$, при сохранении постоянной разницы температур $\Delta T$, приводит к монотонному росту скорости движения наночастиц. Поэтому термофорез углеродных наночастиц на плоской многослойной подложке h-BN будет наиболее сильно проявляться при низких температурах.

\section{Финансирование работы}

Исследование выполнено при финансовой поддержке РФФИ в рамках научного проекта № 18-29-19135. Вычислительные ресурсы предоставлены межведомственным суперкомпьютерным центром РАН.

\section{Конфликт интересов}

Автор заявляет, что у него нет конфликта интересов.

\section{Список литературы}

[1] N. Azong-Wara, C. Asbach, B. Stahlmecke, H. Fissan, H. Kaminski, S. Plitzko, D. Bathen, T.A.J. Kuhlbusch. J. Nanopart. Res. 15, 1530 (2013).

[2] A. Barreiro, R. Rurali, E.R. Hernandez, J. Moser, T. Pichler, L. Forro, A. Bachtold. Science 320, 775-778 (2008).

[3] R. Piazza. Soft Matter 4, 9, 1740-1744 (2008).

[4] B. Liu, K. Zhou. Prog. Mater. Sci. 100, 99-169 (2019).

[5] R. Guerra, U. Tartaglino, A. Vanossi, E. Tosatti Ballistic nanofriction. Nature Mater. 9, 634-637 (2010).

[6] E. Panizon, R. Guerra, E. Tosatti. PNAS 114 (34), E7035 (2017).

[7] M. Jafary-Zadeh, C.D. Reddy, V. Sorkin, Y.-W. Zhang. Nanoscale Res. Lett. 7, 148 (2012).

[8] A.V. Savin, Y.S. Kivshar. Transport of fullerene molecules along graphene nanoribbons. Sci. Rep. 2, 1012 (2012).

[9] M. Becton, X. Wang. J. Chem. Theory Comput. 10, 722 (2014).

[10] R. Rajegowda, S.K. Kannam, R. Hartkamp, S.P. Sathian. Nanotechbology 29, 21, 215401 (2018).

[11] D. Wang, L. Wang, Z. Hu. Nanoscale Res. Lett. 15, 203 (2020).

[12] А. В. Савин, О.И. Савина. ФТТ, 63, 4, 564-571 (2021).

[13] P.A.E. Schoen, J.H. Walther, S. Arcidiacono, D. Poulikakos, P. Koumoutsakos. Nano Lett. 6, 9, 1910 (2006).

[14] P.A.E. Schoen, J.H. Walther, D. Poulikakos, P. Koumoutsakos. Appl. Phys. Lett. 90, 253116 (2007).

[15] J. Shiomi, S. Maruyama. Nanotechnology 20, 055708 (2009).

[16] E. Oyarzua, J.H. Walther, C.M Megaridis, P. Koumoutsakos, H.A. Zambrano. ACS Nano 11, 10, 9997 (2017).
[17] R. Rajegowda, S.K. Kannam, R. Hartkamp, S.P. Sathian. Nanotechnology 28, 155401 (2017).

[18] E. Oyarzua, J.H. Walther, H.A. Zambrano. Phys. Chem. Chem. Phys. 20, 5, 3672 (2018).

[19] Q. Cao. J. Phys. Chem. C 123, 29750 (2019).

[20] A. Panahi, P. Sadeghi, A. Akhlaghi, M.H. Sabour. Diamond \& Related Materials 110108105 (2020).

[21] H.A. Zambrano, J.H. Walther, R.L. Jaffe. J. Chem. Phys. 131, 241104 (2009).

[22] M.V.D. Prasad, B. Bhattacharya. Nano Lett., 16, 4, 2174 (2016).

[23] M.V.D. Prasad, B. Bhattacharya. Nano Lett. 17, 4, 2131 (2017).

[24] R. Rurali, E.R. Hernández. Chem. Phys. Lett. 497, 62 (2010).

[25] N. Wei, H.-Q. Wang, J.-C. Zheng. Nanoscale Res. Lett. 7, 1, 154 (2012).

[26] A.V. Savin, E.A. Korznikova, S.V. Dmitriev, Phys. Rev. B 92, 035412 (2015).

[27] А.В. Савин, Е.А. Корзникова, С.В. Дмитриев, ФТТ 57, 11, 2278 (2015).

[28] A.V. Savin, E.A. Korznikova, S.V. Dmitriev, Phys. Rev. B 99, $235411(2019)$.

[29] J.H. Los, J.M.H. Kroes, K. Albe, R.M. Gordillo, M.I. Katsnelson, A. Fasolino. Phys. Rev. B 96, 184108 (2017).

[30] A.K. Rappé, C.J. Casewit, K.S. Colwell, W.A. Goddard III, W.M. Skiff. J. Am. Chem. Soc. 114, 10024 (1992).

[31] A. Vanossi, N. Manini, M. Urbakh, S. Zapperi, E. Tosatti, Rev. Mod. Phys. 85, 529 (2013).

[32] D. Mandelli, W. Ouyang, O. Hod, M. Urbakh, Phys. Rev. Lett. 122, 076102 (2019).

Редактор Т.Н. Василевская 